\title{
Prevalence of IgG Antibodies to SARS-CoV-2 in Wuhan - Implications for the Longevity of Antibodies Against SARS-CoV-2
}

Tao Liu ( $\sim$ liutaozaiwuda@whu.edu.cn )

Wuhan University Zhongnan Hospital https://orcid.org/0000-0002-4202-8218

\section{Sanyun Wu}

wuhan university

Huangheng Tao

wuhan university

Guang Zeng

Wuhan University

Fuling Zhou

wuhan university

Xinghuan Wang

Wuhan University

\section{Research}

Keywords: Longevity, Antibodies, IgG, SARS-CoV-2

Posted Date: November 2nd, 2020

DOI: https://doi.org/10.21203/rs.3.rs-99748/v1

License: () (1) This work is licensed under a Creative Commons Attribution 4.0 International License.

Read Full License 


\section{Abstract}

\section{Background}

It is to be determined whether people infected with SARS-CoV-2 will develop long-term immunity against SARS-CoV-2 and retain long-lasting antibodies after the infection is resolved. This study was to explore the outcomes of IgG antibodies to SARS-CoV-2 in four groups of individuals in Wuhan, China.

\section{Methods}

We conducted a cross-sectional study on the following four groups who received both COVID-19 $\mathrm{IgM} / \mathrm{IgG}$ tests and RT-PCR tests for SARS-CoV-2 from February 29, 2020 to April 29, 2020: 1470 hospitalized patients with COVID-19 from Leishenshan Hospital, Zhongnan Hospital of Wuhan University, and Wuhan No. 7 Hospital, 3832 healthcare providers without COVID-19 diagnosis, 19555 general workers, and 1616 other patients to be admitted to the hospital $(\mathrm{N}=26473)$. COVID-19 patients who received IgM/lgG tests $<21$ days after symptom onset were excluded.

Results

IgG prevalence was $89.8 \%$ (95\% Cl 88.2-91.3\%) in COVID-19 patients, 4.0\% (95\% Cl 3.4-4.7\%) in healthcare providers, $4.6(95 \% \mathrm{Cl} 4.3-4.9 \%)$ in general workers, and $1.0 \%$ in other patients ( $\mathrm{p}$ all $<0.001 \mathrm{for}$ comparisons with COVID-19 patients). IgG prevalence increased significantly by age among healthcare workers and general workers. Among hospitalized COVID-19 patients, presence of IgG antibodies to SARS-CoV-2 was not associated with most disease severity, presence of comorbidities, treatment received, and clinical characteristics. We identified 24 hospitalized patients with COVID-19 and multiple COVID-19 antibody tests who lost previously detected IgG antibodies to SARS-CoV-2

\section{Conclusions}

IgG antibodies to SARS-CoV-2 in infected people may become undetectable overtime.

\section{Introduction}

Currently, coronavirus disease in 2019 (COVID-19) caused by severe acute respiratory syndrome coronavirus 2 (SARS-CoV-2) ${ }^{1-4}$ has become a global pandemic. The virus was freely transmitted among residents in the communities ${ }^{5-8}$ in Wuhan, China from late November 2019 till several days after the lockdown of the city on January 23, 2020. Most of SARS-CoV-2 infections do not require medical attention $^{9-11}$ and only about $5 \%$ COVID-19 cases in China need intensive care ${ }^{12}$. Numerous therapeutic approaches are under investigation around the world, including existing anti-human immunodeficiency virus (HIV), hepatitis B virus (HBV), hepatitis $C$ virus ( $\mathrm{HCV}$ ) and influenza virus medications ${ }^{13}$, RNAdependent RNA polymerase inhibitor Remdesivir ${ }^{14}$, antimalarials (chloroquine/hydroxychloroquine), IL-6 
blocker Tocilizumab, serum from recovered COVID-19 patients, and inactivated virus, subunit and recombinant vaccines.

The presence of SARS-CoV-2 in COVID-19 patients is usually confirmed using real-time reversetranscriptase polymerase chain reaction (RT-PCR) method. ${ }^{15-17}$ Due to sample collection method, discrepancies in personnel skills training and low virus load in throat swabs after symptom onset ${ }^{18}$, RTPCR methods have high false-negative test results ${ }^{17,19,20}$. Several days after symptom onset when infected patients start to seek medical attention, virus load in clinical specimens of upper respiratory tract will become relatively low ${ }^{18,21}$. It often takes two or three repeatedly collected specimens to get a positive test result in patients. SARS-CoV-2 enters respiratory epithelial cells via interactions with angiotensin converting enzyme 2 (ACE2) ${ }^{22}$. The spike (S) protein of SARS-CoV-2 mediates binding of the virus with Spike protein's receptor ACE2 and promotes fusion of viral and host cell membranes and subsequent virus entry into the COVID-19 host cell. In patients infected with SARS-CoV-2, IgM antibodies are detectable around 7 days post infection and IgG antibodies usually take two weeks to develop. ${ }^{18,22-24}$ Recently, several COVID-19 IgM/IgG rapid tests have been developed around the world ${ }^{25}$. It is reported that in some recovered COVID-19 patients who had two negative RT-PCR tests on nasal or throat swabs taken at least 24 hours apart, segments of virus RNA were still detected in other types of clinical samples, especially in fecal swabs ${ }^{26}$. Additionally, some patients had recurrent positive RT-PCR tests on nasal or throat swabs after recovered from COVID-19. ${ }^{26}$ It still unknown whether COVID-19 patients will develop long-term immunity against SARS-CoV-2 and retain long-lasting antibodies after the infection is resolved. Large-scale sero-epidemiological studies are also needed to assess infection attack rates and disease incidence in the population and herd immunity. In this study, we reported the experiences in COVID-19 $\operatorname{lgM} / \lg$ testing in Wuhan. We assessed prevalence of $\lg G$ antibodies against SARS-CoV- 2 in hospitalized patients with COVID-19 from Zhongnan Hospital of Wuhan University and Leishenshan Hospital (set up on an emergency basis to admit COVID-19 patients and managed by Zhongnan Hospital of Wuhan University), and Wuhan No. 7 Hospital, healthcare providers without a confirmed COVID-19 diagnosis working in Zhongnan Hospital of Wuhan University, and people from the general population in Wuhan. Considering that Wuhan was the early epicenter of COVID-19 outbreak, Zhongnan Hospital of Wuhan University is a major hospital caring for COVID-19 patients and screening for people with COVID-19 symptoms. Prevalence of IgM antibodies to SARS-CoV-2 were also assessed as a secondary outcome. Most of these healthcare providers would be inevitably exposed to SARS-CoV-2 during the early days of the outbreak (from late November 2019 to January 20, 2020) when person-to-person transmission was not suspected and little personal protection against this virus was employed among medical personnel, a large proportion of whom would get infected with the virus.

\section{Methods}

\section{Study Design and Participants}


The study was approved by the institutional ethics board at Zhongnan Hospital of Wuhan University. Requirement for written informed consent was waived by the institutional ethics board for emerging infectious diseases. We conducted a cross-sectional study by including the following four groups of individuals who received both COVID-19 IgM/lgG tests and RT-PCR tests for SARS-CoV-2 from February 29, 2020 to April 29, 2020: hospitalized patients with COVID-19 from Leishenshan Hospital, Zhongnan Hospital of Wuhan University, and Wuhan No. 7 Hospital who received these tests before discharge from hospital, healthcare providers (doctors, nurses, and nursing workers) without a confirmed COVID-19 diagnosis working in Zhongnan Hospital of Wuhan University who received these tests before resuming normal clinical services for patients without COVID-19, general workers without a confirmed COVID-19 diagnosis in Wuhan before returning to work, and other patients without a confirmed COVID-19 diagnosis who received these screening tests before being admitted to Zhongnan Hospital of Wuhan University (Figure 1). There were 1603 hospitalized patients with COVID-19 who received COVID-19 IgM/lgG tests from February 29 to April 5, 2020 (the last test result was used for analyses). Eighty-four patients were transferred from Zhongnan Hospital of Wuhan University to Leishenshan Hospital and were only counted once each. We excluded 133 patients with COVID-19 whose IgM/IgG tests were less than 21 days after symptom onset to allow enough time for IgG antibodies against SARS-CoV-2 to develop. There were 4099 healthcare providers working in Zhongnan Hospital of Wuhan University, of whom 118 were diagnosed of COVID-19 before March 16, 2020 and 3835 healthcare providers without diagnosed COVID-19 received both tests before resuming normal clinical services. Three healthcare providers who were tested positive for SARS-CoV-2 by RT-PCR tests in their throat swabs were also excluded from the analyses. Before returning to work, 19570 general workers without a diagnosis of COVID-19 in Wuhan who received both tests at Zhongnan Hospital of Wuhan University, of whom 15 were tested positive for SARS-CoV-2 by RTPCR tests in their throat swabs and were excluded from the analyses. Before admitted to Zhongnan Hospital of Wuhan University for other conditions, 1628 patients without COVID-19 diagnosis received both tests for screening for SARS-CoV-2, of whom 12 were tested positive for SARS-CoV-2 by RT-PCR tests in their throat swabs and were removed from the analyses. In total, we included 1470 patients with COVID-19, 3832 healthcare providers, 19555 workers, and 1616 other patients in the final analyses $(\mathrm{N}=26473)$.

Among those 118 healthcare workers who were diagnosed of COVID-19 before March 16, 2020, 97 received COVID-19 received IgM/IgG tests during hospitalization or their quarantine. IgG prevalence was $92.8 \%(90 / 97)$ and IgM prevalence was $49.5 \%(48 / 97)$.

Death among hospitalized COVID-19 patients during hospitalization was ascertained from electronic medical records. Two physicians extracted the following data using data collection form from electronic medical records: demographic information such as age and sex, RT-PCR test date and results, COVID-19 $\mathrm{lgM} / \mathrm{IgG}$ test date and results, date of symptom onset for COVID-19 patients, treatments received, and clinical outcomes. Another physician in the research team reviewed the collected data. Main outcome of interest was presence of IgG antibodies to SARS-CoV-2. Presence of IgM antibodies to SARS-CoV-2 was a secondary outcome of interest in this study. 
Diagnosis of COVID-19 was based on epidemiological history, clinical manifestations and presence of SARS-CoV-2 in clinical samples confirmed by using real-time RT-PCR method. ${ }^{11}$ There were changes in diagnosis of COVID-19 in China, and the case definition was gradually broadened to allow for detection of milder cases. ${ }^{57}$ The confirmed cases were estimated to be 4 times less than that if the later broader case definition had been adopted earlier. Severity of status of patients with COVID-19 at admission was defined as moderate, severe, or critical. Patients with mild diseases were not admitted to the above three hospitals and were generally admitted to Fangcang Hospitals (makeshift hospitals).

\section{RT-PCR test for SARS-CoV-2 virus RNA}

Clinical specimens were collected for RT-PCR test for SARS-CoV-2. Clinical specimens in COVID-19 patients included nasal swabs, throat swabs, sputum, anal swabs, and bronchoalveolar lavage (BAL), and clinical specimens in the other three groups of people without confirmed COVID-19 diagnosis were only throat swabs. In brief, clinical specimens were collected from these people by trained nurses or physicians wearing proper personal protection equipment. RT-PCR tests for SARS-CoV-2 were performed using a nucleic acid detection kit following the manufacturer's protocol. The lower limit of detection (LOD) for the RT-PCR test is 500 copies/ml. The test simultaneously amplifies and detects two target genes, including open reading frame $1 \mathrm{ab}$ (ORF1ab) and nucleocapsid protein (N). Primers used for those two target genes are as follows: ORF1ab: forward primer CCCTGTGGGTTTTACACTTAA, reverse primer ACGATTGTGCATCAGCTGA; and the probe 5'-VIC-CCGTCTGCGGTATGTGGAAAGGTTATGG-BHQ1-3'; N: forward primer GGGGAACTTCTCCTGCTAGAAT, reverse primer CAGACATTTTGCTCTCAAGCTG, and the probe 5'-FAM- TTGCTGCTGCTTGACAGATT-TAMRA-3'. Conditions for the amplifications were incubation at $50{ }^{\circ} \mathrm{C}$ for 15 minutes and $95^{\circ} \mathrm{C}$ for 5 minutes, followed by 40 cycles of denaturation at $94{ }^{\circ} \mathrm{C}$ for 15 seconds and extension at $55^{\circ} \mathrm{C}$ for 45 seconds. The diagnostic criteria for positive and negative RT-PCR results were based on the recommendation by the National Institute for Viral Disease Control and Prevention (China): positive result $<37$ cycle threshold value (Ct-value) and negative result $\geq 40$. A Ctvalue of 37-39 required retesting.

\section{COVID-19 IgM/lgG test for antibodies against SARS-CoV-2}

Serum samples from these people were collected. Methods for testing serum IgM and IgG antibodies to SARS-CoV-2 were previously described. ${ }^{58}$ COVID-19 IgM/lgG test kits contained recombinant SARS-CoV-2 antigens (spike protein and nucleocapsid protein) labelled with magnetic beads (tested on a fully-automated chemiluminescence immunoassay analyzer) or colloidal gold (test card), anti-human IgM monoclonal antibody, and anti-human IgG monoclonal antibody. These test kits were reported to

have high sensitivity and specificity ${ }^{25,58}$. According to the manufacturers, the sensitivity and specificity are $\sim 90 \%$ and $>99 \%$ for $\operatorname{lgM}$, and $\sim 98 \%$ and $\sim 98 \%$ for lgG, respectively. Validation studies of these test kits conducted by the Department of Laboratory Medicine at Zhongnan Hospital of Wuhan University showed that the sensitivity and specificity are $~ 80 \%$ and $~ 99 \%$ for $\operatorname{lgM}$, and $\sim 96 \%$ and $\sim 98 \%$ for $\operatorname{lgG}$, respectively. 


\section{Statistical Analysis}

Continuous variables were reported using mean and 95\% confidence interval (Cl) if normally distributed. Days from symptom onset to $\mathrm{lgM} / \mathrm{lg}$ test were reported as median and interquartile because this variable was not normally distributed. Age was handled as both continuous variable and categorical variable (by 10 years of age) in the analyses. Categorical variables were described as frequency rates and percentages. The $\chi 2$ test was used for the comparison of categorical variables and Fisher's exact test was used when frequency was too low. Multigroup comparisons of age between the four groups were performed using ANOVA test, following by Tukey test for adjusting for multiple comparisons. Prevalence of positive IgG test results and $95 \% \mathrm{Cl}$ was also reported. Logistic regression models were fitted and $\chi 2$ tests were used to compare seroprevalence of IgG and IgM antibodies to SARS-CoV-2 between the four groups. For the assessment of IgM/lgG test results in hospitalized patients, the last test result for each patient was used. We only included individuals from the four groups who received both COVID-19 $\mathrm{lgM} / \mathrm{lgG}$ tests and RT-PCR tests for SARS-CoV-2. Individuals with missing data were excluded from the analyses. Sensitivity analyses were performed in subgroups, such as age groups and different sexes. Statistical analyses were conducted using SAS software version 9.4 (SAS Institute; Carey, NC). A 2-sided $p$ value of $<0.05$ was considered statistically significant.

\section{Results}

\section{Characteristics of participants}

Mean age was 58.7 years in 1470 COVID-19 patients, 37.1 years in 3832 healthcare providers, 41.6 years in 19555 general workers and 53.3 years in other patients (Table 1). Hospitalized COVID-19 patients were composed of more older people, while healthcare providers and general workers were mostly young adults. Among COVID-19 patients, median time from symptom onset to $\mathrm{lgM} / \mathrm{lg}$ test was 41 days (interquartile range 33-50 days). The last RT-PCR tests for SARS-CoV-2 were positive in only eight COVID19 patients and all these eight patients were tested positive for IgG antibodies to SARS-CoV-2.

\section{Prevalence of IgG antibodies to SARS-CoV-2}

Prevalence of IgG antibodies to SARS-CoV-2 was 89.8\% (95\% Cl 88.2-91.3\%) in COVID-19 patients (Table 2) compared to $4.0 \%(95 \% \mathrm{Cl} 3.4-4.7 \%)$ in healthcare providers, $4.6 \%(95 \% \mathrm{Cl} 4.3-4.9 \%)$ in general workers, and $1.0 \%$ in other patients ( $p$ all $<0.001$ for comparing to COVID-19 patients). Only the comparison of IgG prevalence between healthcare workers and general workers was not significant $(p=0.39)$. IgG prevalence increased significantly by age among healthcare providers, and was $2.8 \%$ in those $<30$ years old, $9.6 \%$ in those $60-69$ years old and $10.0 \%$ in those $\geq 70$ years old ( $p<0.001$ for trend). IgG prevalence also increased significantly by age among general workers.

\section{IgG antibodies to SARS-CoV-2 and mortality in COVID-19 patients}


Among COVID-19 patients, mean age was similar between those with IgG antibodies to SARS-CoV-2 and those without (Table 3). Presence of IgG antibodies to SARS-CoV-2 was not associated with most demographic characteristics, disease severity, presence of comorbidities, treatment received, and clinical characteristics, except for antibiotics treatment, chloroquine/hydroxychloroquine treatment, and needing intubation. IgG prevalence among hospitalized patients with COVID-19 by demographic and clinical characteristics are presented in Table 4. Case mortality rate was 1.3\% (95\% CI 0.7-1.9\%) in those with IgG antibodies to SARS-CoV-2 during hospitalization, similar to $3.3 \%$ (95\% $\mathrm{Cl} 0.4-6.2 \%$ ) in those without.

\section{IgG Antibody to SARS-CoV-2 degradation}

We identified 24 hospitalized patients with COVID-19 and multiple COVID-19 antibody tests who lost previously detected lgG antibodies to SARS-CoV-2 (Table 5). These patients all had positive tests for IgG antibodies to SARS-CoV-2. However, their most recent tests for IgG antibodies to SARS-CoV-2 were negative. Mean age of these patients were 56.6 years old and $58.3 \%$ were male. All these patients had moderate COVID-19 at admission, 3 of whom were progressed to critical status. Seven (29.2\%) of these patients had hypertension, and $3(12.5 \%)$ had diabetes.

\section{Prevalence of IgM antibodies to SARS-CoV-2}

Prevalence of IgM antibodies to SARS-CoV-2 was 31.4\% (95\% Cl 29.0-33.7\%) in COVID-19 patients, $1.5 \%$ (95\% Cl 1.1-1.8\%) in healthcare providers, 1.3 (95\% Cl 1.1-1.5\%) in general workers, and 0.2\% (95\% Cl 0$0.4 \%$ ) in other patients (Table 6).

\section{Discussion}

We analyzed prevalence of IgG antibodies to SARS-CoV-2 in hospitalized COVID-19 patients, healthcare providers without a confirmed COVID-19 diagnosis, general workers, and other patients to be admitted to hospital. The most intriguing finding of this study is that we found in a subpopulation of hospitalized patients with COVID-19 who tested positive for IgG antibodies to SARS-CoV-2 that the IgG antibodies to SARS-CoV-2 became undetectable overtime. It seems that the longevity of the IgG antibodies to SARSCoV-2 is questionable. Our findings are consistent with previous reports on the longevity of antibodies to SARS-CoV-2. ${ }^{27-29}$ Infected patients with no symptoms ${ }^{27}$ or mild symptoms ${ }^{28,29}$ experienced rapid decay of antibodies to SARS-CoV-2 in a couple of months.

The observed only 4\% prevalence of IgG antibodies to SARS-CoV-2 in healthcare providers without confirmed COVID-19 also raised the possibility of fast decay of these antibodies. Zhongnan Hospital of Wuhan University is a major hospital caring for COVID-19 patients and screening for people with COVID19 symptoms in the early epicenter of this pandemic. Most of these healthcare providers in our study would be inevitably exposed to SARS-CoV-2 during the early days of the outbreak (from late November 2019 to January 20, 2020) when person-to-person transmission was not suspected and little personal protection against this virus was employed among medical personnel. Two months of daily work with high-volume patient intake but no proper personal protection equipment is more than sufficient to expect 
a rather high proportion of SARS-CoV-2 infection among these healthcare providers. In Zhongnan Hospital of Wuhan University, 2.88\% (118/4099) healthcare workers were diagnosed with COVID-19 before March 16, 2020. Seropositivity results consistently showed that only a small portion of infected patients were detected by RT-PCR tests. ${ }^{30-34}$ With a moderate estimation, the true infection rate would be $\sim$ ten times that had been confirmed, i.e., $>25 \%$ of those healthcare providers without diagnosed COVID19 had been infected. However, only $4 \%$ of those healthcare providers without diagnosed COVID-19 tested positive for IgG antibodies to SARS-CoV-2. A plausible explanation would be the lack of persistent antibodies.

Our observed high prevalence of IgG antibodies to SARS-CoV-2 in older groups (60-69 years old and $\geq 70$ years old) among health care workers and general worker in Wuhan also raised the concern about the longevity of IgG antibodies to SARS-CoV-2, as young or middle-aged people usually took more social responsibilities and had higher chances to get infected during lockdown of the city. We also found that $>10 \%$ of confirmed COVID-19 cases had no detectable serum levels of IgG antibodies to SARS-CoV-2 after 21 days post symptom onset. They were unlikely to not produce IgG antibodies to SARS-CoV-2 after being infected with the virus ${ }^{18,22-24,35}$. Long et al reported that after 17-19 days post symptom onset, IgG was positive in all patients with COVID-19. ${ }^{35}$ Lack of blood samples $>17$ days post symptom onset may be responsible for negative IgG tests in some patients reported in previous literatures. ${ }^{23,} 24$ In our hospitalized patients, all of them should have produced IgG antibodies to the virus. Those $>10 \%$ patients who had no IgG antibodies after 21 days post symptom onset most likely lost those IgG antibodies after the infection was resolved.

Why antibodies to SARS-CoV-2 in infected persons fade so quickly unlike antibodies to SARS-CoV-1? After infection with SARS-CoV-1, patients start to produce SARS-specific IgG antibody in the second week, which persists for a long time ${ }^{36,37}$. Even after 210 days after symptom onset, neutralizing viral antibodies (anti-viral IgG) are still detectable in recovered SARS patients ${ }^{38}$. It is believed that the Spike protein and nucleocapsid protein play a central role in the antibody production ${ }^{36,37,39}$. Research has focused on developing vaccines and therapeutics targeting the Spike protein ${ }^{40,41}$ and the nucleocapsid protein $^{42}$. However, our findings indicate that people are unlikely to develop long-lasting antibodies to SARS-CoV-2 after SARS-CoV-2 infection. Infections with some viruses, such as HIV, do not illicit robust protective immunity ${ }^{43}$, while common cold coronavirus only generate partial protective immunity ${ }^{44}$. Cross-reactive antibodies in convalescent SARS patients' sera can neutralize other human betacoronaviruses ${ }^{45}$, as those viruses share a significant B-cell epitope overlapping the heptad repeat-2 region of the Spike protein. SARS-CoV-2 also belongs to betacoronaviruses. However, no populations had shown explicit immunity to SARS-CoV-2 and people were generally susceptible to this virus before it first jumped from wild animals to humans. ${ }^{22,46-50}$ It might be due to the dramatic differences between SARSCoV-1 and SARS-CoV-2 in the receptor-binding region of Spike protein and the key amino acid residues involved in the interaction with human ACE2. ${ }^{22}$ Neutralizing antibodies to SARS-CoV-1 may not produce reliable immunoprotection against SARS-CoV-2. However, why long-lasting antibodies are not produced 
after SARS-CoV-2 infection is still to be studied. It also reported that antibody titer seems to be higher in patients with severe COVID-19 diseases and lower in asymptomatic patients and patients with mild symptoms. ${ }^{27-29,51} \mathrm{~A}$ recent study characterized the viral spike protein receptor-binding domain-specific monoclonal antibodies derived from single B cells of patients with COVID-19. ${ }^{51}$ In that study, the three most server cases (one dead) had much higher plasma binding activities to SARS-CoV-2 spike protein, spike protein receptor-binding domain, and nucleocapsid protein than the other five cases with mild symptoms, which casts some doubts on the relationship between antibody response and disease progression and the utilization of neutralizing antibodies as prophylactic and therapeutic SARS-CoV-2 interventions. We also found mortality risks were similar between hospitalized COVID-19 patients with IgG antibodies to SARS-CoV-2 and those without, which indicates that absence of IgG antibodies may not affect clinical end outcome. Whether therapeutic antibodies targeting the spike protein are effective will also be a question. Antibody-dependent enhancement of viral entry via the binding of those neutralizing antibodies and the spike protein is also a big concern to be closely monitored ${ }^{52-55}$.

COVID-19 IgM/lgG tests in the US and around the world as reported in the news constantly showed that the true infection rate would be 10 to 80 times higher than that had been confirmed by RT-PCR tests for SARS-CoV-2. ${ }^{30-34}$ Seroprevalence of antibodies to SARS-CoV-2 in 1021 people before resuming work from April 3 to 15, 2020 in Wuhan was reported to be $~ 10 \%,{ }^{30}$ about 20 times higher than the infection attack rate calculated from the confirmed COVID-19 cases. In Los Angeles County, California, the seroprevalence of IgM/lgG antibodies to SARS-CoV-2 was $4.65 \%$, over 40 times higher than that estimated from confirmed cases. ${ }^{31}$ In New York City, a record $21.2 \%$ positive rate was reported with young and middleaged people having the highest positive rate. Our observed $4.6 \%$ prevalence of IgG antibodies to SARSCoV-2 in general workers is also about 10 times higher than that of the general population in Wuhan (50,000 cases of COVID-19 in 11 million people). The proportion of people infected with SARS-CoV- 2 who have no symptom or only mild symptoms that do not need medical attention or hospitalization may account for the majority of SARS-CoV-2 infections. Detecting patients with SARA-CoV-2 infection who are not in urgent need of hospitalization may have important public health applications in tracing their close contacts and preventing these people from spreading the infection to others. Pool testing to enable largescale screening ${ }^{56}$ and introducing antibody test into the testing scheme may be considered to detect most of the infected persons.

The main strength of this study is that we analyzed data on COVID-19 IgM/lgG tests on a large cohort of individuals from three hospitals in Wuhan, the epicenter of COVID-19 outbreak in China. Antibody tests on healthcare providers provided valuable information on the longevity of antibodies to SARS-CoV-2 after infection. Limitations of this study include that we used convenient samples from three hospitals. We also do not have long-term follow-up data on recovered COVID-19 patients. Whether or not those patients will lose COVID-19 IgG antibodies in the next few months is still to be studied. Additionally, COVID-19 IgM/IgG test does not have perfect sensitivity and specificity for detecting IgG antibodies to SARS-CoV-2 in the serum. Nevertheless, we observed that long-lasting antibodies to SARS-CoV-2 are unlikely produced after infection. Our findings may have important implications for herd immunity, antibody-based 
therapeutics, public health strategies, surveillance of past infections of SARS-COV-2, and vaccine development.

In conclusion, we found in a small sample of hospitalized patients with COVID-19 who tested positive for IgG antibodies to SARS-CoV-2 that these antibodies became undetectable overtime. In addition, very few healthcare providers without confirmed COVID-19 diagnosis in Wuhan have IgG antibodies to SARS-CoV2 , though a substantial portion of them had been infected with the virus. About $90 \%$ hospitalized COVID19 patients still have IgG antibodies to SARS-CoV-2 before discharge from hospital, while more than $10 \%$ of COVID-19 patients do not have those antibodies after 21 days post symptom onset. Antibodies against this virus produced in infected people seem to be not long lasting.

\section{Conclusion}

IgG antibodies to SARS-CoV-2 in infected people may become undetectable overtime.

\section{Declarations}

\section{Ethical Approval and Consent to participate}

The study was approved by the institutional ethics board at Zhongnan Hospital of Wuhan University. Requirement for written informed consent was waived by the institutional ethics board for emerging infectious diseases.

\section{Consent for publication}

The authors have not published any related papers from the same study, and this paper is not under consideration elsewhere. None of this paper's contents have been previously published. All authors have read and approved the manuscript.

\section{Availability of supporting data}

For protection of patient privacy, data and analyses codes are available from the corresponding authors on request. All request for raw and analyzed data and materials will be reviewed by the corresponding authors to verify whether the request is subject to any intellectual property or confidentiality obligations. Access will be granted after a signed data access agreement is attained.

\section{Competing interests}

The authors have no conflicts of interest to report.

\section{Primary Funding Sources}

Part of the study was supported by National Key Research and Development Program of China (2020YFC0845500) and Postdoctoral Research Foundation of China (2020M670071ZX). The content is 
solely the responsibility of the authors and does not necessarily represent the official views of the sponsors.

\section{Role of the Funder/Sponsor}

The sponsors had no role in the design and conduct of the study; collection, management, analysis, and interpretation of the data; and preparation, review, or approval of the manuscript, and decision to submit the manuscript for publication.

\section{Financial Disclosures}

The authors have no conflict of interest to disclose.

\section{Author Contributions}

Dr. X. Wang had full access to all the data in the study and takes responsibility for the integrity of the data and the accuracy of the data analysis. Drs T. Liu, S. Wu and H. Tao contributed equally to the study. Drs T. Liu, F. Zhou, and X. Wang contributed equally as senior authors. Concept and design: F. Zhou and X. Wang. Acquisition, analysis, or interpretation of data: T. Liu, S. Wu and H. Tao, Guang Zeng. Drafting of the manuscript: T Liu, S. Wu. Critical revision of the manuscript for important intellectual content: S. Wu, X. Wang. Statistical analysis: T. Liu, H. Tao.

\section{Acknowledgements}

Not applicable.

\section{References}

1. Zhu N, Zhang D, Wang W, et al. A Novel Coronavirus from Patients with Pneumonia in China, 2019. N Eng/ J Med. Jan 2020;doi:10.1056/NEJMoa2001017

2. Chen N, Zhou M, Dong X, et al. Epidemiological and clinical characteristics of 99 cases of 2019 novel coronavirus pneumonia in Wuhan, China: a descriptive study. Lancet. Jan 2020;doi:10.1016/S01406736(20)30211-7

3. Lu R, Zhao X, Li J, et al. Genomic characterisation and epidemiology of 2019 novel coronavirus: implications for virus origins and receptor binding. Lancet. Jan 2020;doi:10.1016/S01406736(20)30251-8

4. Viruses CSGotICoTo. The species Severe acute respiratory syndrome-related coronavirus: classifying 2019-nCoV and naming it SARS-CoV-2. Nat Microbiol. Mar 2020;doi:10.1038/s41564-020-0695-z

5. Chan JF, Yuan S, Kok KH, et al. A familial cluster of pneumonia associated with the 2019 novel coronavirus indicating person-to-person transmission: a study of a family cluster. Lancet. Jan 2020;doi:10.1016/S0140-6736(20)30154-9 
6. Rothe C, Schunk M, Sothmann P, et al. Transmission of 2019-nCoV Infection from an Asymptomatic Contact in Germany. N Engl J Med. Jan 2020;doi:10.1056/NEJMc2001468

7. Li Q, Guan X, Wu P, et al. Early Transmission Dynamics in Wuhan, China, of Novel CoronavirusInfected Pneumonia. N Engl J Med. Jan 2020;doi:10.1056/NEJMoa2001316

8. Phan LT, Nguyen TV, Luong QC, et al. Importation and Human-to-Human Transmission of a Novel Coronavirus in Vietnam. N Engl J Med. Jan 2020;doi:10.1056/NEJMc2001272

9. Li LQ, Huang T, Wang YQ, et al. 2019 novel coronavirus patients' clinical characteristics, discharge rate and fatality rate of meta-analysis. J Med Virol. Mar 2020;doi:10.1002/jmv.25757

10. Jiang F, Deng L, Zhang L, Cai Y, Cheung CW, Xia Z. Review of the Clinical Characteristics of Coronavirus Disease 2019 (COVID-19). J Gen Intern Med. Mar 2020;doi:10.1007/s11606-020-05762w

11. Deng SQ, Peng HJ. Characteristics of and Public Health Responses to the Coronavirus Disease 2019 Outbreak in China. J Clin Med. Feb 2020;9(2)doi:10.3390/jcm9020575

12. Guan WJ, Ni ZY, Hu Y, et al. Clinical Characteristics of Coronavirus Disease 2019 in China. N Eng/ J Med. Feb 2020;doi:10.1056/NEJMoa2002032

13. Li G, De Clercq E. Therapeutic options for the 2019 novel coronavirus (2019-nCoV). Nat Rev Drug Discov. Mar 2020;19(3):149-150. doi:10.1038/d41573-020-00016-0

14. Holshue ML, DeBolt C, Lindquist S, et al. First Case of 2019 Novel Coronavirus in the United States. $N$ Engl J Med. 03 2020;382(10):929-936. doi:10.1056/NEJMoa2001191

15. Huang C, Wang Y, Li X, et al. Clinical features of patients infected with 2019 novel coronavirus in Wuhan, China. Lancet. Jan 2020;doi:10.1016/S0140-6736(20)30183-5

16. Wang D, Hu B, Hu C, et al. Clinical Characteristics of 138 Hospitalized Patients With 2019 Novel Coronavirus-Infected Pneumonia in Wuhan, China. JAMA. Feb 2020;doi:10.1001/jama.2020.1585

17. Wang W, Xu Y, Gao R, et al. Detection of SARS-CoV-2 in Different Types of Clinical Specimens. JAMA. Mar 2020;doi:10.1001/jama.2020.3786

18. Wölfel R, Corman VM, Guggemos W, et al. Virological assessment of hospitalized patients with COVID-2019. OriginalPaper. Nature. 2020-04-01 2020:1-10. doi:doi:10.1038/s41586-020-2196-x

19. Pan Y, Zhang D, Yang P, Poon LLM, Wang Q. Viral load of SARS-CoV-2 in clinical samples. Lancet Infect Dis. Feb 2020;doi:10.1016/S1473-3099(20)30113-4

20. Peiris JS, Chu CM, Cheng VC, et al. Clinical progression and viral load in a community outbreak of coronavirus-associated SARS pneumonia: a prospective study. Lancet. May 2003;361(9371):176772. doi:10.1016/s0140-6736(03)13412-5

21. Zou L, Ruan F, Huang M, et al. SARS-CoV-2 Viral Load in Upper Respiratory Specimens of Infected Patients. N Eng/ J Med. Feb 2020;doi:10.1056/NEJMc2001737

22. Zhou $P$, Yang $X L$, Wang $X G$, et al. A pneumonia outbreak associated with a new coronavirus of probable bat origin. Nature. Mar 2020;579(7798):270-273. doi:10.1038/s41586-020-2012-7 
23. Zhao J, Yuan Q, Wang H, et al. Antibody responses to SARS-CoV-2 in patients of novel coronavirus disease 2019. Clin Infect Dis. Mar 28 2020;doi:10.1093/cid/ciaa344

24. Xiang F, Wang $X$, He X, et al. Antibody Detection and Dynamic Characteristics in Patients with COVID19. Clin Infect Dis. Apr 19 2020;doi:10.1093/cid/ciaa461

25. Li Z, Yi Y, Luo X, et al. Development and Clinical Application of A Rapid IgM-lgG Combined Antibody Test for SARS-CoV-2 Infection Diagnosis. J Med Virol. Feb 27 2020;doi:10.1002/jmv.25727

26. Lan L, Xu D, Ye G, et al. Positive RT-PCR Test Results in Patients Recovered From COVID-19. JAMA. Feb 2020;doi:10.1001/jama.2020.2783

27. Long QX, Tang XJ, Shi QL, et al. Clinical and immunological assessment of asymptomatic SARSCoV-2 infections. Nat Med. Jun 2020;doi:10.1038/s41591-020-0965-6

28. Ibarrondo FJ, Fulcher JA, Goodman-Meza D, et al. Rapid Decay of Anti-SARS-CoV-2 Antibodies in Persons with Mild Covid-19. letter. The New England Journal of Medicine. 2020-07-21 2020;doi:NJ202007213830004

29. Seow J, Graham C, Merrick B, et al. Longitudinal evaluation and decline of antibody responses in SARS-CoV-2 infection. medRxiv. 2020-07-11 2020;doi:10.1101/2020.07.09.20148429

30. Wu X, Fu B, Chen L, Feng Y. Serological tests facilitate identification of asymptomatic SARS-CoV-2 infection in Wuhan, China. J Med Virol. Apr 20 2020;doi:10.1002/jmv.25904

31. Sood N, Schaeffer Center for Health Policy and Economics SPSoPP, University of Southern California, Los Angeles, Simon P, et al. Seroprevalence of SARS-CoV-2-Specific Antibodies Among Adults in Los Angeles County, California, on April 10-11, 2020. JAMA. 2020;323(23):2425-2427. doi:10.1001/jama.2020.8279

32. S S, A W, G P, et al. Seroprevalence of anti-SARS-CoV-2 IgG Antibodies in Geneva, Switzerland (SEROCoV-POP): A Population-Based Study. Lancet (London, England). 06/11/2020 2020;doi:10.1016/S0140-6736(20)31304-0

33. X X, J S, S N, et al. Seroprevalence of Immunoglobulin M and G Antibodies Against SARS-CoV-2 in China. Nature medicine. 06/05/2020 2020;doi:10.1038/s41591-020-0949-6

34. Havers FP, Reed C, Lim T, et al. Seroprevalence of Antibodies to SARS-CoV-2 in 10 Sites in the United States, March 23-May 12, 2020. JAMA Intern Med. Jul 2020;doi:10.1001/jamainternmed.2020.4130

35. Long Q-X, Liu B-Z, Deng H-J, et al. Antibody responses to SARS-CoV-2 in patients with COVID-19. OriginalPaper. Nature Medicine. 2020-04-29 2020:1-4. doi:doi:10.1038/s41591-020-0897-1

36. Zhu M. SARS Immunity and Vaccination. Cell Mol Immunol. Jun 2004;1(3):193-8.

37. Ho MS, Chen WJ, Chen HY, et al. Neutralizing antibody response and SARS severity. Emerg Infect Dis. Nov 2005;11(11):1730-7. doi:10.3201/eid1111.040659

38. Shi Y, Wan Z, Li L, et al. Antibody responses against SARS-coronavirus and its nucleocaspid in SARS patients. J Clin Virol. Sep 2004;31(1):66-8. doi:10.1016/j.jcv.2004.05.006

39. Du L, He Y, Zhou Y, Liu S, Zheng BJ, Jiang S. The spike protein of SARS-CoV--a target for vaccine and therapeutic development. Nat Rev Microbiol. Mar 2009;7(3):226-36. doi:10.1038/nrmicro2090 
40. ter Meulen J, van den Brink EN, Poon LL, et al. Human monoclonal antibody combination against SARS coronavirus: synergy and coverage of escape mutants. PLoS Med. Jul 2006;3(7):e237. doi:10.1371/journal.pmed.0030237

41. Liu J, Shao H, Tao Y, et al. Production of an anti-severe acute respiratory syndrome (SARS) coronavirus human monoclonal antibody Fab fragment by using a combinatorial immunoglobulin gene library derived from patients who recovered from SARS. Clin Vaccine Immunol. May 2006;13(5):594-7. doi:10.1128/cvi.13.5.594-597.2006

42. Zhao A, Qin W, Han Y, et al. Isolation and identification of an scFv antibody against nucleocapsid protein of SARS-CoV. Microbes Infect. Jul 2007;9(8):1026-33. doi:10.1016/j.micinf.2007.04.008

43. M A, TM A, XG Y, et al. HIV-1 Superinfection Despite Broad CD8+ T-cell Responses Containing Replication of the Primary Virus. Nature. 11/28/2002 2002;420(6914)doi:10.1038/nature01200

44. KA C, HF P, M S, DA T. The Time Course of the Immune Response to Experimental Coronavirus Infection of Man. Epidemiology and infection. 1990 Oct 1990;105(2)doi:10.1017/s0950268800048019

45. Chan KH, Chan JF, Tse H, et al. Cross-reactive antibodies in convalescent SARS patients' sera against the emerging novel human coronavirus EMC (2012) by both immunofluorescent and neutralizing antibody tests. J Infect. Aug 2013;67(2):130-40. doi:10.1016/j.jinf.2013.03.015

46. Kissler SM, Tedijanto C, Goldstein E, Grad YH, Lipsitch M. Projecting the transmission dynamics of SARS-CoV-2 through the postpandemic period. Science. 05 2020;368(6493):860-868. doi:10.1126/science.abb5793

47. Wu JT, Leung K, Leung GM. Nowcasting and forecasting the potential domestic and international spread of the 2019-nCoV outbreak originating in Wuhan, China: a modelling study. The Lancet. doi:10.1016/S0140-6736(20)30260-9

48. Lam TT, Jia N, Zhang YW, et al. Identifying SARS-CoV-2-related coronaviruses in Malayan pangolins. Nature. 07 2020;583(7815):282-285. doi:10.1038/s41586-020-2169-0

49. Xiao K, Zhai J, Feng Y, et al. Isolation of SARS-CoV-2-related coronavirus from Malayan pangolins. Nature. 07 2020;583(7815):286-289. doi:10.1038/s41586-020-2313-x

50. Shang J, Ye G, Shi K, et al. Structural basis of receptor recognition by SARS-CoV-2. Nature. 05 2020;581(7807):221-224. doi:10.1038/s41586-020-2179-y

51. Ju B, Zhang Q, Ge J, et al. Human neutralizing antibodies elicited by SARS-CoV-2 infection. Nature. May 2020;doi:10.1038/s41586-020-2380-z

52. Walls AC, Xiong X, Park YJ, et al. Unexpected Receptor Functional Mimicry Elucidates Activation of Coronavirus Fusion. Cell. Feb 21 2019;176(5):1026-1039.e15. doi:10.1016/j.cell.2018.12.028

53. Wan Y, Shang J, Sun S, et al. Molecular Mechanism for Antibody-Dependent Enhancement of Coronavirus Entry. J Virol. Feb 14 2020;94(5)doi:10.1128/jvi.02015-19

54. Cao X. COVID-19: immunopathology and its implications for therapy. Nat Rev Immunol. Apr 2020;doi:10.1038/s41577-020-0308-3 
55. Iwasaki A, Yang Y. The potential danger of suboptimal antibody responses in COVID-19.

BriefCommunication. Nature Reviews Immunology. 2020-04-21 2020:1-3. doi:doi:10.1038/s41577020-0321-6

56. Cherif A, Grobe N, Wang X, Kotanko P. Simulation of Pool Testing to Identify Patients With Coronavirus Disease 2019 Under Conditions of Limited Test Availability. JAMA Netw Open. 06 2020;3(6):e2013075. doi:10.1001/jamanetworkopen.2020.13075

57. TK T, P W, Y L, EHY L, GM L, BJ C. Effect of Changing Case Definitions for COVID-19 on the Epidemic Curve and Transmission Parameters in Mainland China: A Modelling Study. The Lancet Public health. 2020 May 2020;5(5)doi:10.1016/S2468-2667(20)30089-X

58. Zeng H, Department of Laboratory Medicine ZHoWU, Wuhan, China, Xu C, et al. Antibodies in Infants Born to Mothers With COVID-19 Pneumonia. JAMA. 2020;doi:10.1001/jama.2020.4861

\section{Tables}

\section{Table 1. Characteristics of hospitalized COVID-19 patients, healthcare providers without confirmed COVID-19, general workers, and other patients in Wuhan $(n=26473)$.}

\begin{tabular}{|c|c|c|c|c|c|}
\hline & \multicolumn{4}{|c|}{ Mean (95\% Confidence Interval) or $\mathrm{n}(\%)$} & \multirow{2}{*}{$\begin{array}{l}P \\
\text { value }\end{array}$} \\
\hline & $\begin{array}{l}\text { COVID-19 } \\
\text { Patients }\end{array}$ & $\begin{array}{l}\text { Healthcare } \\
\text { Providers }\end{array}$ & $\begin{array}{l}\text { General } \\
\text { Workers }\end{array}$ & $\begin{array}{l}\text { Other } \\
\text { Patients }\end{array}$ & \\
\hline & $(n=1470)$ & $(n=3832)$ & $(n=19555)$ & $(n=1616)$ & \\
\hline Age (years) & $58.7(58.0-59.4)$ & $37.1(36.7-37.4)$ & $\begin{array}{l}41.6(41.4- \\
41.8)\end{array}$ & $\begin{array}{l}53.3(52.4- \\
54.2)\end{array}$ & $<0.001$ \\
\hline \multicolumn{6}{|l|}{$\begin{array}{l}\text { Age group } \\
\text { (years) }\end{array}$} \\
\hline$<30$ & $30(2.0)$ & $1236(32.3)$ & $3960(20.3)$ & $154(9.5)$ & \multirow[t]{6}{*}{$<0.001$} \\
\hline $30-39$ & $130(8.8)$ & $1265(33.0)$ & $6068(31.0)$ & $166(10.3)$ & \\
\hline $40-49$ & $203(13.8)$ & $607(15.8)$ & $3688(18.9)$ & $239(14.8)$ & \\
\hline $50-59$ & $356(24.2)$ & $600(15.7)$ & $4031(20.6)$ & $459(28.4)$ & \\
\hline $60-69$ & $430(29.3)$ & $114(3.0)$ & $1315(6.7)$ & $357(22.1)$ & \\
\hline$\geq 70$ & $321(21.8)$ & $10(0.3)$ & $493(2.5)$ & $241(14.9)$ & \\
\hline \multicolumn{6}{|l|}{ Sex } \\
\hline Female & $745(50.7)$ & $2596(67.7)$ & $9735(49.8)$ & $708(43.8)$ & \multirow[t]{2}{*}{$<0.001$} \\
\hline Male & $725(49.3)$ & $1236(32.3)$ & $9820(50.2)$ & $908(56.2)$ & \\
\hline
\end{tabular}


COVID-19 patients: hospitalized patients with COVID-19 pneumonia from Leishenshan Hospital, Zhongnan Hospital of Wuhan University, and Wuhan No. 7 Hospital who received both COVID-19 IgM/lgG tests and RT-PCR tests before discharge from hospital.

Healthcare providers: doctors, nurses, and nursing workers without a confirmed COVID-19 diagnosis working in Zhongnan Hospital of Wuhan University who received both COVID-19 IgM/lgG tests and RTPCR tests before resuming normal clinical services for patients without COVID-19.

General workers: general workers in Wuhan who received both COVID-19 IgM/lgG tests and RT-PCR tests before returning to work.

Other patients who received both COVID-19 IgM/lgG tests and RT-PCR tests to screen for SARS-CoV-2 infections before being admitted to Zhongnan Hospital of Wuhan University.

Table 2. Prevalence of IgG antibodies to SARS-CoV-2 among hospitalized COVID-19 patients, healthcare providers without confirmed COVID-19, general workers, and other patients in Wuhan $(n=26473)$.

\begin{tabular}{|lllll|}
\hline \multicolumn{5}{|c|}{ Prevalence \% $(95 \%$ Confidence Interval) } \\
& COVID-19 Patients & Healthcare Providers & General Workers & Other patients \\
\hline & $(\mathrm{n}=1470)$ & $(\mathrm{n}=3832)$ & $(\mathrm{n}=19555)$ & $(\mathrm{n}=1616)$ \\
\hline All & $89.8(88.2-91.3)$ & $4.0(3.4-4.7)$ & $4.6(4.3-4.9)$ & $1.0(0.5-1.5)$ \\
\hline Age group (years) & & & & \\
\hline$<30$ & $90.0(78.6-100.0)$ & $2.8(1.9-3.8)$ & $3.5(2.9-4.1)$ & 0 \\
\hline $30-39$ & $89.2(83.8-94.6)$ & $3.8(2.7-4.8)$ & $4.7(4.2-5.3)$ & $0.6(0.0-1.8)$ \\
\hline $40-49$ & $92.1(88.4-95.9)$ & $4.4(2.8-6.1)$ & $4.6(3.9-5.3)$ & $1.3(0.0-2.7)$ \\
\hline $50-59$ & $92.1(89.3-94.9)$ & $5.5(3.7-7.3)$ & $4.8(4.1-5.4)$ & $0.7(0.0-1.4)$ \\
\hline $60-69$ & $89.1(86.1-92.0)$ & $9.6(4.1-15.2)$ & $6.8(5.4-8.1)$ & $1.1(0.0-2.2)$ \\
\hline$\geq 70$ & $86.9(83.2-90.6)$ & $10.0(0.0-32.6)$ & $5.9(3.8-8.0)$ & $2.1(0.3-3.9)$ \\
\hline Sex & & & & $1.0(0.3-1.7)$ \\
\hline Female & $90.3(88.2-92.5)$ & $3.7(3.0-4.4)$ & $5.0(4.5-5.4)$ & $1.0(0.3-1.6)$ \\
\hline Male & $89.2(87.0-91.5)$ & $4.8(3.6-6.0)$ & $4.3(3.9-4.7)$ & \\
\hline
\end{tabular}

Table 3. Demographic and clinical characteristics of hospitalized patients with COVID-19 pneumonia from Leishenshan Hospital, Zhongnan Hospital of Wuhan University, and Wuhan No. 7 Hospital $(n=1470)$. 


\begin{tabular}{|c|c|c|c|}
\hline & \multicolumn{3}{|c|}{ Mean (95\% Confidence Interval) or $\mathrm{n}(\%)$} \\
\hline & Positive & Negative & P Value \\
\hline & $(n=1320)$ & $(n=150)$ & \\
\hline Age (years) & $58.5(57.8-59.2)$ & $60.8(58.3-63.2)$ & 0.061 \\
\hline \multicolumn{4}{|l|}{ Age group (years) } \\
\hline$<30$ & $27(2.0)$ & $3(2.0)$ & \multirow[t]{6}{*}{0.26} \\
\hline $30-39$ & $116(8.8)$ & $14(9.3)$ & \\
\hline $40-49$ & $187(14.2)$ & $16(10.7)$ & \\
\hline $50-59$ & $328(24.8)$ & $28(18.7)$ & \\
\hline $60-69$ & $383(29.0)$ & $47(31.3)$ & \\
\hline$\geq 70$ & $279(21.1)$ & $42(28.0)$ & \\
\hline \multicolumn{4}{|l|}{ Sex } \\
\hline Female & $673(51.0)$ & $72(48.0)$ & \multirow[t]{2}{*}{0.49} \\
\hline Male & $647(49.0)$ & $78(52.0)$ & \\
\hline \multicolumn{4}{|c|}{ Severity of COVID-19 at admission } \\
\hline Moderate & $1004(76.1)$ & $108(72.0)$ & \multirow[t]{3}{*}{0.51} \\
\hline Severe & $263(19.9)$ & $34(22.7)$ & \\
\hline Critical & $53(4.0)$ & $8(5.3)$ & \\
\hline \multicolumn{4}{|l|}{ Comorbidities } \\
\hline Any & $739(56.0)$ & $87(58.0)$ & 0.64 \\
\hline Hypertension & $390(29.5)$ & $50(33.3)$ & 0.34 \\
\hline Cardiovascular disease (any) & $418(31.7)$ & $55(36.7)$ & 0.21 \\
\hline Cerebrovascular disease & $40(3.0)$ & $7(4.7)$ & 0.32 \\
\hline Diabetes & $165(12.5)$ & $23(15.3)$ & 0.32 \\
\hline Chronic bronchitis/asthma & $23(1.7)$ & $2(1.3)$ & 0.71 \\
\hline \multicolumn{4}{|l|}{ Treatment received } \\
\hline Anti-virus & $594(45.0)$ & $65(43.3)$ & 0.7 \\
\hline Antibiotics & $566(42.9)$ & $91(60.7)$ & 0.001 \\
\hline Corticosteroids & $148(11.2)$ & $18(12.0)$ & 0.77 \\
\hline
\end{tabular}




\begin{tabular}{|llll|}
\hline Chloroquine/hydroxychloroquine & $123(9.3)$ & $6(4.0)$ & 0.029 \\
\hline Immunoglobulin & $216(16.4)$ & $22(14.7)$ & 0.59 \\
\hline Traditional Chinese Medicine & $12(0.9)$ & $2(1.3)$ & 0.64 \\
\hline Clinical characteristics & $1142(86.5)$ & $121(80.7)$ & 0.051 \\
\hline Progression to critical status & $71(5.6)$ & $13(9.2)$ & 0.09 \\
\hline ICU admission & $76(5.8)$ & $14(9.3)$ & 0.084 \\
\hline Need intubation & $28(2.1)$ & $11(7.3)$ & 0.001 \\
\hline Need Ventilator & $71(5.4)$ & $11(7.3)$ & 0.32 \\
\hline Death & $17(1.3)$ & $5(3.3)$ & 0.065 \\
\hline
\end{tabular}

ICU: Intensive Care Unit.

* 61 patients with a critical status at admission were excluded.

Table 4. Prevalence of IgM and IgG antibodies to SARS-CoV-2 among hospitalized patients with COVID19 from Leishenshan Hospital, Zhongnan Hospital of Wuhan University, and Wuhan No. 7 Hospital $(n=1470)$. 


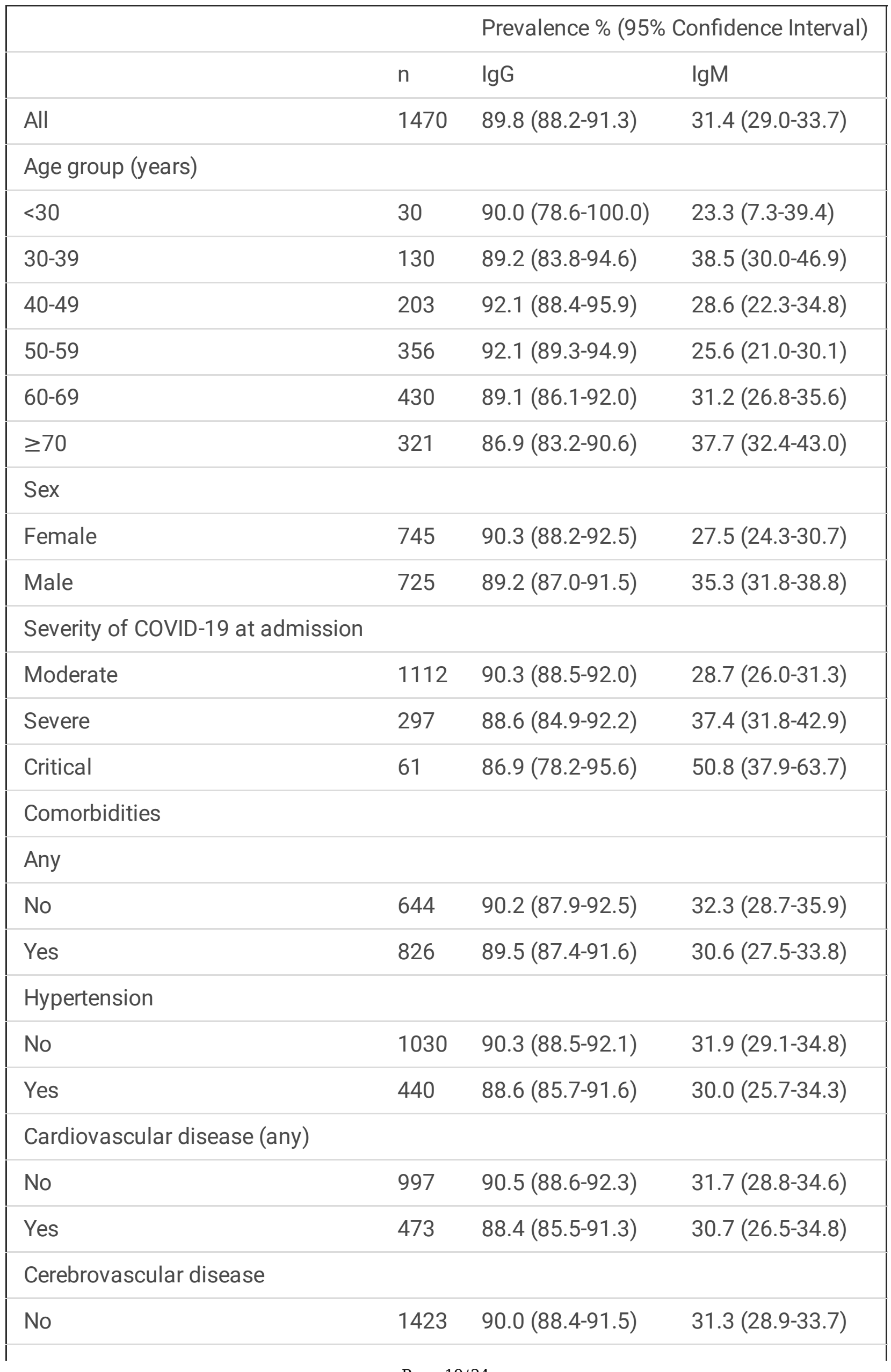




\begin{tabular}{|c|c|c|c|}
\hline Yes & 47 & $85.1(74.5-95.7)$ & $34.0(20.0-48.1)$ \\
\hline \multicolumn{4}{|c|}{ Diabetes } \\
\hline No & 1282 & $90.1(88.5-91.7)$ & $31.1(28.6-33.7)$ \\
\hline Yes & 188 & $87.8(83.0-92.5)$ & $33.0(26.2-39.8)$ \\
\hline \multicolumn{4}{|c|}{ Chronic bronchitis/asthma } \\
\hline No & 1445 & $89.8(88.2-91.3)$ & $31.2(28.8-33.6)$ \\
\hline Yes & 25 & $92.0(80.6-100.0)$ & $40.0(19.4-60.6)$ \\
\hline \multicolumn{4}{|c|}{ Treatment received } \\
\hline \multicolumn{4}{|c|}{ Anti-virus } \\
\hline No & 811 & $89.5(87.4-91.6)$ & $31.6(28.4-34.8)$ \\
\hline Yes & 659 & $90.1(87.9-92.4)$ & $31.1(27.6-34.7)$ \\
\hline \multicolumn{4}{|c|}{ Antibiotics } \\
\hline No & 813 & $92.7(91.0-94.5)$ & $30.1(27.0-33.3)$ \\
\hline Yes & 657 & $86.1(83.5-88.8)$ & $32.9(29.3-36.5)$ \\
\hline \multicolumn{4}{|c|}{ Corticosteroids } \\
\hline No & 1304 & $89.9(88.2-91.5)$ & $29.1(26.7-31.6)$ \\
\hline Yes & 166 & $89.2(84.4-93.9)$ & $48.8(41.1-56.5)$ \\
\hline \multicolumn{4}{|c|}{ Chloroquine/hydroxychloroquine } \\
\hline No & 1341 & $89.3(87.6-90.9)$ & $30.8(28.3-33.3)$ \\
\hline Yes & 129 & $95.3(91.7-99.0)$ & $37.2(28.8-45.7)$ \\
\hline \multicolumn{4}{|c|}{ Vitamin C } \\
\hline No & 1232 & $89.6(87.9-91.3)$ & $30.4(27.8-32.9)$ \\
\hline Yes & 238 & $90.8(87.0-94.5)$ & $36.6(30.4-42.7)$ \\
\hline \multicolumn{4}{|c|}{ Immunoglobulin } \\
\hline No & 1456 & $89.8(88.3-91.4)$ & $30.8(28.5-33.2)$ \\
\hline Yes & 14 & $85.7(64.7-100.0)$ & $85.7(64.7-100.0)$ \\
\hline \multicolumn{4}{|c|}{ Traditional Chinese Medicine } \\
\hline No & 207 & $86.0(81.2-90.8)$ & $35.3(28.7-41.8)$ \\
\hline Yes & 1263 & $90.4(88.8-92.0)$ & $30.7(28.2-33.3)$ \\
\hline
\end{tabular}




\begin{tabular}{|lccc|}
\hline Clinical characteristics & & & \\
\hline Progression to critical status & \\
No & & & \\
\hline Yes & 1325 & $90.3(88.7-91.9)$ & $30.0(27.5-32.4)$ \\
\hline ICU admission & 84 & $84.5(76.6-92.4)$ & $38.1(27.5-48.7)$ \\
\hline No & & & \\
\hline Yes & 1380 & $90.1(88.6-91.7)$ & $30.5(28.1-32.9)$ \\
\hline Need intubation & 90 & $84.4(76.8-92.1)$ & $44.4(34.0-54.9)$ \\
\hline No & & & \\
\hline Yes & 1431 & $90.3(88.8-91.8)$ & $30.8(28.4-33.2)$ \\
\hline Need Ventilator & 39 & $71.8(57.0-86.6)$ & $51.3(34.9-67.7)$ \\
\hline No & & & \\
\hline Yes & 1388 & $90.0(88.4-91.6)$ & $30.6(28.2-33.0)$ \\
\hline Death & 82 & $86.6(79.1-94.1)$ & $43.9(32.9-54.9)$ \\
\hline No & & & \\
\hline Yes & 1448 & $90.0(88.4-91.5)$ & $31.0(28.6-33.4)$ \\
\hline
\end{tabular}

ICU: Intensive Care Unit.

a 61 patients with a critical status at admission were excluded.

Table 5. Hospitalized patients with COVID-19 who lost previously detected IgG antibodies to SARS-CoV-2 $(n=24)$. 


\begin{tabular}{|c|c|c|}
\hline & \multicolumn{2}{|c|}{ Days from symptom onset ${ }^{a}$} \\
\hline & IgG Positive & IgG Negative \\
\hline Patient 1 & 49 & 55,59 \\
\hline Patient 2 & 30 & 34,35 \\
\hline Patient 3 & 38 & 49 \\
\hline Patient 4 & 59 & 69 \\
\hline Patient 5 & 31 & 42 \\
\hline Patient 6 & 40 & 50 \\
\hline Patient 7 & 53 & 64 \\
\hline Patient 8 & 41 & 51 \\
\hline Patient 9 & 42 & $56,61,66$ \\
\hline Patient 10 & 62,63 & 69 \\
\hline Patient 11 & 42 & 53 \\
\hline Patient 12 & 21 & 27 \\
\hline Patient 13 & 35 & 41 \\
\hline Patient 14 & 44 & 50 \\
\hline Patient 15 & 38 & 44 \\
\hline Patient 16 & 38 & 44 \\
\hline Patient 17 & 43 & 49,53 \\
\hline Patient 18 & 45 & 49 \\
\hline Patient 19 & 38 & 42 \\
\hline Patient 20 & 26 & 28 \\
\hline Patient 21 & 37 & 39 \\
\hline Patient 22 & 33 & 35 \\
\hline Patient 23 & 47 & 50 \\
\hline Patient 24 & 49 & 54 \\
\hline
\end{tabular}

a Days from symptom onset to COVID-19 antibody test. 
Table 6. Prevalence of IgM antibodies to SARS-CoV-2 among hospitalized COVID-19 patients, healthcare providers without confirmed COVID-19, general workers, and other patients in Wuhan ( $n=26473)$.

\begin{tabular}{|lllll|}
\hline \multicolumn{5}{|l}{ Prevalence \% (95\% Confidence Interval) } \\
& COVID-19 Patients & Healthcare Providers & General Workers & Other patients \\
& $(\mathrm{n}=1470)$ & $(\mathrm{n}=3832)$ & $(\mathrm{n}=19555)$ & $(\mathrm{n}=1616)$ \\
\hline All & $31.4(29.0-33.7)$ & $1.5(1.1-1.8)$ & $1.3(1.1-1.5)$ & $0.2(0.0-0.4)$ \\
\hline Age group (years) & & & & \\
\hline$<30$ & $23.3(7.3-39.4)$ & $1.1(0.5-1.6)$ & $0.7(0.4-1.0)$ & 0 \\
\hline $30-39$ & $38.5(30.0-46.9)$ & $1.2(0.6-1.8)$ & $1.0(0.7-1.2)$ & 0 \\
\hline $40-49$ & $28.6(22.3-34.8)$ & $2.1(1.0-3.3)$ & $1.7(1.3-2.1)$ & $0.4(0.0-1.2)$ \\
\hline $50-59$ & $25.6(21.0-30.1)$ & $2.0(0.9-3.1)$ & $1.7(1.3-2.1)$ & 0 \\
\hline $60-69$ & $31.2(26.8-35.6)$ & $2.6(0.0-5.6)$ & $1.7(1.0-2.5)$ & $0.6(0.0-1.3)$ \\
\hline$\geq 70$ & $37.7(32.4-43.0)$ & 0 & $2.4(1.1-3.8)$ & 0 \\
\hline Sex & & & & $0.1(0.0-0.4)$ \\
\hline Female & $27.5(24.3-30.7)$ & $1.5(1.0-2.0)$ & $1.7(1.4-1.9)$ & $0.2(0.0-0.5)$ \\
\hline Male & $35.3(31.8-38.8)$ & $1.4(0.7-2.0)$ & $0.9(0.7-1.1)$ & \\
\hline
\end{tabular}

\section{Figures}




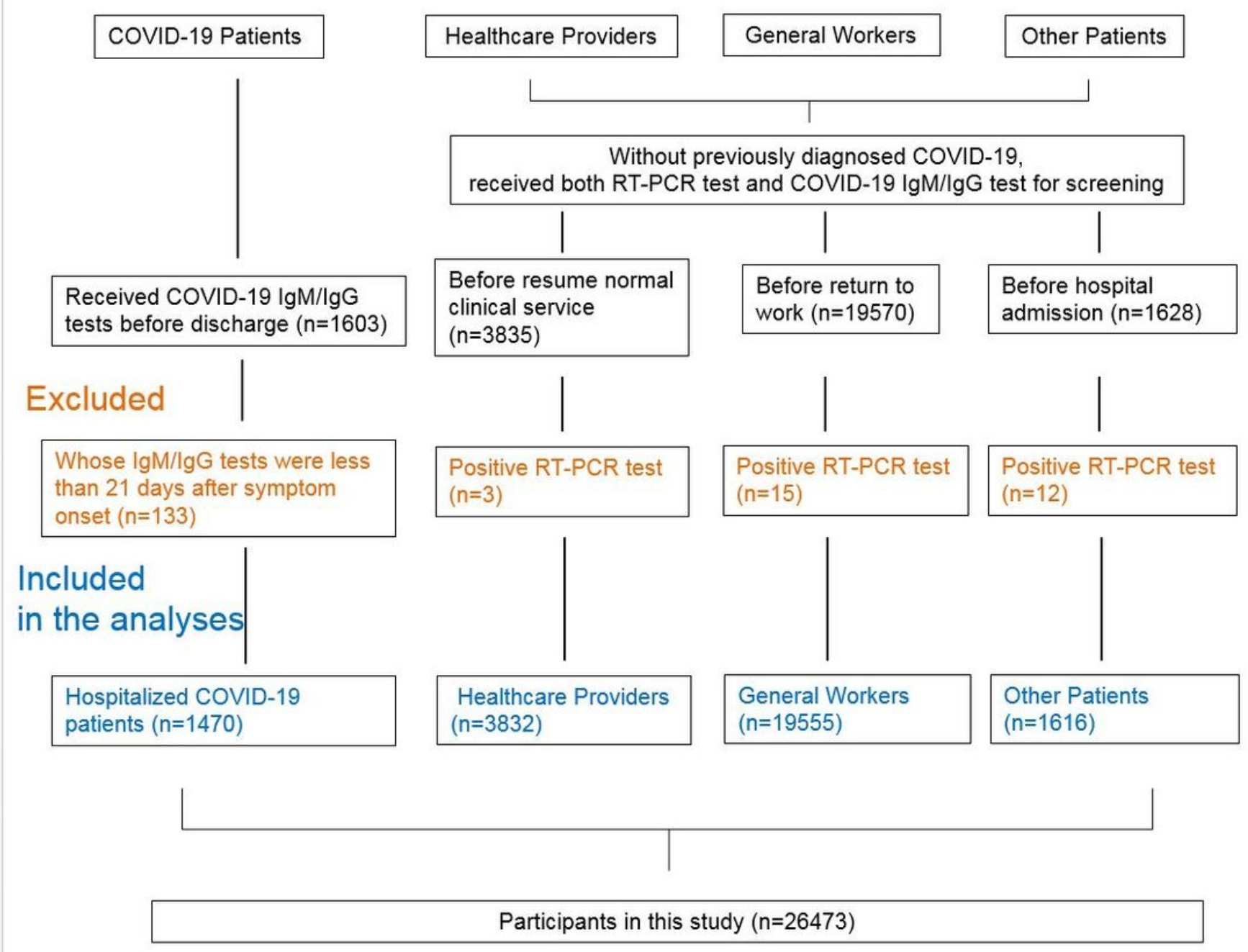

\section{Figure 1}

Flowchart of the numbers of participants included in the analyses. COVID-19 patients: hospitalized patients with COVID-19 from Leishenshan Hospital, Zhongnan Hospital of Wuhan University, and Wuhan No. 7 Hospital who received both COVID-19 IgM/lgG tests and RT-PCR tests before discharge from hospital. Healthcare providers: doctors, nurses, and nursing workers without a confirmed COVID-19 diagnosis working in Zhongnan Hospital of Wuhan University who received both COVID-19 IgM/lgG tests and RT-PCR tests before resuming normal clinical services for patients without COVID-19. General workers: general workers without a confirmed COVID-19 diagnosis in Wuhan who received both COVID-19 IgM/lgG tests and RT-PCR tests before returning to work. Other patients: patients without a confirmed COVID-19 diagnosis who received both COVID-19 IgM/lgG tests and RT-PCR tests to screen for SARS-CoV2 infections before being admitted to Zhongnan Hospital of Wuhan University. 